Communications in Physics, Vol. 14, No. 2 (2004), pp. 95-99

\title{
BINDING ENERGY OF EXCITON IN QUANTUM DOTS WITH THE CENTRAL-CELL CORRECTION DEPENDING ON THE DOT SIZES
}

\author{
TO THI THAO AND NGUYEN AI VIET \\ Institute of Physics \& Electronics, VAST
}

\begin{abstract}
The binding energy of exciton in quantum dots with a parabolic confinement potential was calculated by variational methods beyond the Kohn-Luttinger effective mass theory, when the central-cell correction was taken into account.We have assumed that a short range potential with two parameters for strength and range for exciton, representing the center-cell effect also depends on dot size. Our result is in good agreement with experiment.
\end{abstract}

\section{INTRODUCTION}

Semiconductor nano particles-quantum dots have been fabricated and extensively investigated in both experimental and theoretical sides. The quantum dots are such small structure as quasi zero-dimensional with strong confinement in all directions. The electrons and holes in quantum dots are fully quantized in a discrete spectrum of energy levels. The strong optical efficiency observed in quantum dots makes them promising candidates for optolectronic and nano devices $[1,2]$.

One well known that the exciton play an important role in determining the optical properties of system. The study of exciton states in quantum dots is a relevant aspect to which many theoretical works have been devoted $[3,4,5,6,7,8,9,10]$.

The effective mass theory provides a simple theoretical model to calculate binding energies of excitons in quantum dots $[3,4,5]$. According to this model, the exciton problem is a hydrogen atom embedded in a diecletric medium with a mass renormalization (effective mass) for the electron-hole pair. While the binding energy of exciton in big quantum dots are described quite accurately, there are large deviation for the binding of small quantum dots from those predicted by this effective mass theory. This Coulombic potential $\left(e^{2} / \epsilon_{0}\right)$ assumed in the breakdown of concept of the static dielectric constant and a correction can be effective by using proper screening function $\epsilon(r)$ approaching $\epsilon_{0}$ as $r \rightarrow \infty$.

Recently some authors $[11,12]$ have studied the central-cell corrections for donors in semiconductors as analogy problem of excitons. Beyond the effective mass theory, instead of the Coulombic potential they have assumed a short range potential with two parameters for the strength and the range for donors, representing the central-cell effects. In this work, using that idea we investigate the problem of excitons in small quantum dots.

Assuming the same two parameters model potential like in the central-cell corrections problem, we will show that the binding energies of excitons increase with reducing of the dot radius $\mathrm{R}$. 


\section{MODEL HAMILTONIAN}

Let us consider the conduction band and valence band are spherical with effective masses $m_{e}^{*}$ and $m_{h}^{*}$ for the electron and hole. The Hamiltonian for an exciton in spherical quantum dots is defined as

$$
H_{x}=E_{g}+\frac{\overrightarrow{p_{e}^{2}}}{2 m_{e}^{*}}+\frac{\overrightarrow{p_{h}^{2}}}{2 m_{h}^{*}}+V_{\text {conf }}\left(\overrightarrow{r_{e}}, \overrightarrow{r_{h}}\right)+U_{c}\left(\overrightarrow{r_{e}}-\overrightarrow{r_{h}}\right)
$$

in which $E_{g}$ is the band gap, $V_{\text {conf }}\left(\overrightarrow{r_{e}}, \overrightarrow{r_{h}}\right)$ is the confinement potential for the electron and hole, which can be calculated approximately by use a simple one-parameter parabolic potential $[4,5]$

$$
V_{\text {conf }}\left(\overrightarrow{r_{e}}, \overrightarrow{r_{h}}\right)=\frac{\omega_{c}^{2}}{2}\left(m_{e}^{*} r_{e}^{2}+m_{h}^{*} r_{h}^{2}\right)
$$
Eq. (8).

The parameter $\omega_{c}$ will be chosen later by changing the radius of quantum dot in

The last term of equation (1) $U_{c}\left(\overrightarrow{r_{e}}-\overrightarrow{r_{h}}\right)$ is the parameter effective potential representing the deviation from the effective mass theory, which is

$$
U_{c}(\vec{r})=-\frac{e^{2}}{\epsilon r}\left[1+\frac{V_{0}}{R^{4 / 3}} \exp \left(-\frac{r^{2}}{\lambda^{2}}\right)\right]
$$

where $\epsilon$ is the static dielectric constant, $\mathrm{R}$ is dot radius, $V_{0}$ and $\lambda$ are parameters representing the strength and the range of the potential in the central-cell region, resp.

The crucial property of the parabolic potential is that the Hamiltonian (1) is separable upon introducing the central-of-mass and relative transformations for the coordinate and momentum $[5,6]$. The Hamiltonian of central-of-mass motion is identified to be the well known oscillator one with the lowest energy level $E_{R O}=\frac{3}{2} \hbar \omega_{c}$. The Hamiltonian of the relative motion equals

$$
H_{r}=\frac{p^{2}}{2 \mu}+V_{c}(r)+U_{c}(r)
$$

where $\mu=m_{e}^{*} m_{h}^{*} /\left(m_{e}^{*}+m_{h}^{*}\right)$ is the reduced mass of the electron-hole pair, $\vec{r}=\overrightarrow{r_{e}}-\overrightarrow{r_{h}}$, and $V_{c}(r)$ is confinement potential for the relative motion

$$
V_{c}(r)=\frac{1}{2} \mu \omega_{c}^{2} r^{2}
$$

Choosing the exciton Rydberg energy $E_{R}=\mu e^{4} / 2 \hbar^{2} \epsilon^{2}$ as the unit of energy and $a_{x}=\frac{\epsilon \hbar^{2}}{\mu e^{2}}$ as the unit of length, the Hamiltonian $H_{r}$ in dimensionless form can be written as

$$
H=\triangle+\frac{\gamma^{2}}{9} \xi^{2}-\frac{2}{\xi}\left(1+v e^{-\alpha \xi^{2}}\right)
$$

where $\xi$ is the dimensionless variable; $v, \alpha, \gamma$ are the dimensionless parameters:

$$
\xi=\frac{r}{a_{x}}, \quad \alpha=\frac{a_{x}^{2}}{\lambda^{2}}, \quad \gamma=\frac{3 \hbar \omega_{c}}{2 E_{R}}, v=\frac{V_{0}}{R^{4 / 3}} .
$$


We will present our results in term of the unit of energy $E_{R}$.

\section{II.1. Binding energy of exciton without the correction}

When the interaction between the electron and hole is omitted, we have a simple relation between the parameter $\omega_{c}$ and dot size $\mathrm{R}$ as

$$
\omega_{c}=\frac{\hbar \pi^{2}}{3 \mu} \frac{1}{R^{2}}
$$

In case of $V_{0}=0$, the potential $U_{c}$ in (3) approaches a simple screened Coulomb potential. Using a trial function

$$
\psi_{0}(\xi)=N_{0} e^{-\frac{1}{4 a_{0}} \xi^{2}},
$$

where $a_{0}$ is the variation parameter and $N_{0}$ is the normalization constant. From (6) and (9) we obtain

$$
<H>_{0}=\frac{3}{4 a_{0}^{2}}-\frac{2 \sqrt{\frac{2}{\pi}}}{a_{0}}+\frac{1}{3} a_{0}^{2} \gamma^{2} .
$$

The variational parameter $a_{0}$ can be obtained by demanding $\partial<H>_{0} / \partial a_{0}=0$. The dot sizes are changed by parameter $\gamma$ (see (7) and (8)). Minimizing $<H>_{0}$ with respect to $a_{0}$, the minimum exciton energy was found. Our result is presented in Fig. 1. This result is similar to the one of other authors $[1,2,3]$.

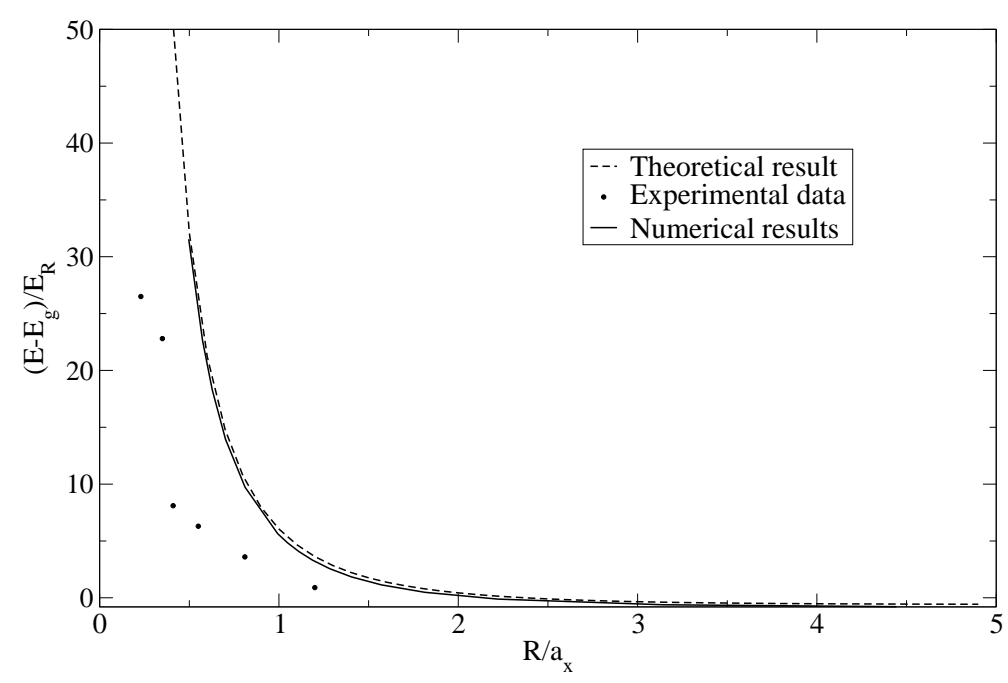

Fig. 1. The dashed line is the spherical confinement potential model result [11], the solid line is our result and the dots are experiment result[1]. 
For comparison, we also plotted these values obtained by using the spherical confinement potential model $[1,2]$

$$
E_{e x}=\frac{\hbar^{2} \pi^{2}}{2 R^{2}}\left[\frac{1}{m_{e}}+\frac{1}{m_{h}}\right]-\frac{1.786 e^{2}}{\epsilon R}-0.248 E_{R},
$$

where $m_{e}$ and $m_{h}$ are effective masses of electron and hole, $\mathrm{R}$ is radius of quantum dot, $\epsilon$ is dielectric constant and $E_{R}$ is bulk exciton binding energy.

\section{II.2. Binding energy of exciton with the correction}

In this section, we study the contribution of the beyond effective mass theory correction $V_{0}$. Using a trail wave function as in equation (9)

$$
\psi_{0}(\xi)=N e^{-\frac{1}{4 a} \xi^{2}},
$$

where $a$ and $N$ is the dimensionless variational parameter and normalization constant, resp. The expectation value of Hamiltonian (6) becomes

$$
<H>=\frac{1}{2\left(a^{2}+2 a^{4} \alpha\right)}\left[-24 a \sqrt{\frac{2}{\pi}}(1+v)+8 a^{6} \gamma^{2} \alpha+9\left(1+2 a^{2} \alpha\right)+4 a^{3}\left(a \gamma^{2}-12 \sqrt{\frac{2}{\pi}} \alpha\right)\right],
$$

here $v, \alpha$ and $\gamma$ and have the same meaning as in (7).

Similarly, the dot size can be changed by changing $\gamma$. In other words, the center-cell effect can be described by changing the dot size R.

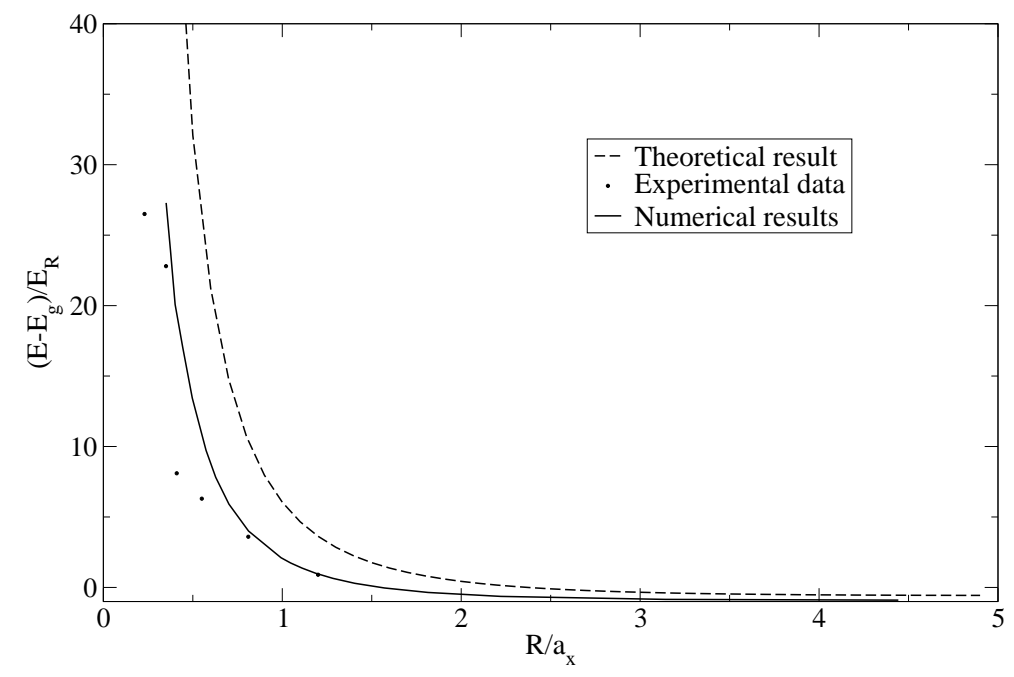

Fig. 2. The dashed line and the solid line is the spherical confinement potential model result [11] and our result, respectively. The dots are experimental result [1].

The minimum exciton energy, $E-E_{g}$, was found by minimizing the expectation value successively with respect to $a$. In the unit of $E_{R}$, the energy of exciton versus the 
parameter $V_{0}$ and $\lambda$ is presented in Fig. 2. Note that the correction enhances the binding energy of exciton.

In summary, we study a present simple model for excitons, which are confined in a quantum dot, when the deviations from effective mass theory was taken into account by using a modified Coulomb potential like in [11]. The binding energy of exciton can be calculated by using $E_{b}=\gamma-<H>_{\min }$, where $<H>_{\min }$ is expressed in units of the Rydberg energy $E_{R}$. It is presented in Fig. 3. The central-cell correction enhances the binding energy of exciton considerably.

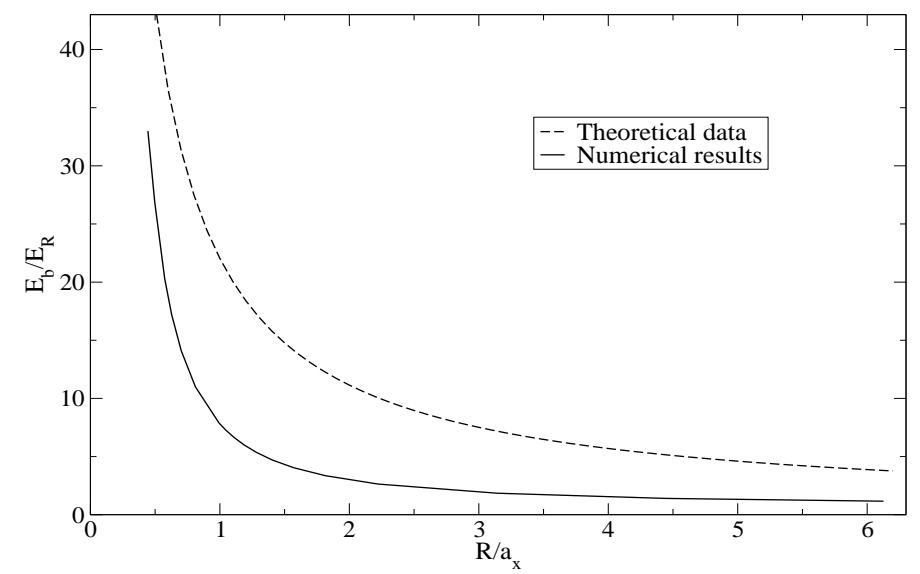

Fig. 3. The dashed line is the spherical confinement potential model result [11], the solid line is our result.

\section{ACKNOWLEDGMENT}

This work is supported by the National program on Basics Research 4.1.1601: "Theory of low dimensional systems and nano-structures".

\section{REFERENCES}

1. L. Banyain, J. S. Koch, Semiconductor Quantum Dots, World-Scientific, 1993.

2. U. Woggon, Optical Properties of Semiconductor Quantum Dots, Springer Verlag, 1997.

3. L. E. Bruss, J. Chem. Phys., 80 (1994) 4403; 90 (1986) 2255.

4. S. V. Nair, S. Sinha, R. C. Ruestagi, Phys. Rev., B35 (1984) 4098.

5. M. E. Said, Semiconductor Sci. Tech., 9 (1994) 272.

6. A, Wixforth et al, Semicond. Sci. Tech., 9 (1994) 215.

7. N. Q. Huong, J. L. Bimman, J. Chem. Phys., 108 (1998) 1769.

8. A. L. Efros et al, Phys. Rev., 54 (1996) 4883.

9. N. T. V. Oanh, N. A. Viet, Int. Mod. Phys. Rev., 62 (2000).

10. N. H. Quang, S. Ohnuma, A. Natori, Phys. Rev., 62 (2000).

11. S. G. Jayama, K. Navaneethakrishnan, J. App. Phys., 89 (2001) 6198.

12. R. J. Heron et al, J. App. Phys., 85 (1999) 893. 
